\title{
Training Cataract Surgeons
}

\author{
Gopal Lingam \\ Sankara Nethralaya, Chennai, India
}

Cataract surgery remains the most common eye surgery performed anywhere in the world. Over the years, the techniques of cataract surgery have become well established, with the standard of care being phacoemulsification with foldable intraocular lens insertion. However, considering the large number of cataract surgeries that are performed throughout the world, even a low incidence of surgery-induced complications gets translated into a considerable number. For sustained delivery of quality eye care to society at large, there is a continuous need to train young ophthalmologists in the correct way of performing cataract surgery. In an article published in this issue of Asian Journal of Ophthalmology, Dubey et al have compared the cataract surgery outcomes performed by trainee registrars with that of senior registrars and consultants. ${ }^{1}$ It is heartening to note from this study that the incidence of complications in the hands of the trainees is not high. The practice of allocating cases to surgeons based on their experience (consultant, senior registrar, or junior registrar) and the anticipated difficulty in performing that particular surgery is a well-accepted procedure. The study by Dubey et al gives evidence to the soundness of this strategy in getting the best results following cataract surgery. ${ }^{1}$ The authors have also shown that despite complications, the visual outcomes were uniformly good in all the groups - evidence to the fact that the complications have been well managed.

Training ophthalmologists in cataract surgery is important, but is also a delicate affair, spawning extensive literature. Gauba et al have described a human reliability analysis of cataract surgery. ${ }^{2}$ They describe a very objective method that identifies the technical errors in performing specific steps of phacoemulsification. Recorded videos of the surgical procedures were evaluated according the predetermined standards. This critical analysis revealed that the more experienced surgeons made fewer errors.

Muhtaseb et al have described a scoring system that enabled the preoperative stratification of patients with cataract according to the anticipated risk of complications. ${ }^{3}$ This weighted scoring system enabled appropriate selection of cases for trainee instruction. A similar system has also been devised by Habib et al ${ }^{4}$ and validated by Osborne et al. ${ }^{5}$

Considering that cataract surgery is done under local anaesthesia with patients remaining awake, supervision needs considerable subtlety. Ideal training programs resulting in the smallest complication rates should have the following components:

- initial training on virtual systems before operating on patients

- selection of uncomplicated cases

- evaluation of the competence of trainee surgeons at periodic intervals using structured formats to give them an opportunity to correct themselves.

\section{References}

1. Dubey R, Chan K, Lertsumitkul S, Grigg J, McCluskey PJ. Cataract surgery outcomes in New South Wales, Australia. Asian J Ophthalmol. 2011;12:124-9.

2. Gauba V, Tsangaris P, Tossounis C, Mitra A, McLean C, Saleh GM. Human reliability analysis of cataract surgery. Arch Ophthalmol. 2008;126:173-7.

3. Muhtaseb M, Kalhoro A, Lonides A. A system for preoperative stratification of cataract patients according to risk of intraoperative complications: a prospective analysis of 1441 cases. $\mathrm{Br} \mathrm{J}$ Ophthalmol. 2004;88:1242-6.

4. Habib MS, Bunce CV, Fraser SG. The role of case mix in the relation of volume and outcome in phacoemulsification. $\mathrm{Br} \mathrm{J}$ Ophthalmol. 2005;89:1143-6.

5. Osborne SA, Severn P, Bunce CV, Fraser SG. The use of a preoperative scoring system for the prediction of phacoemulsification case difficulty and the selection of appropriate cases to be performed by trainees. BMC Ophthalmology. 2006;6:38. 